\title{
Analytical solution for the steady states of the driven Hubbard model
}

\author{
J. Tindall $\odot,{ }^{1, *}$ F. Schlawin $\odot,{ }^{2,3}$ M. A. Sentef $\odot,{ }^{3}$ and D. Jaksch ${ }^{1}$ \\ ${ }^{1}$ Clarendon Laboratory, University of Oxford, Parks Road, Oxford OX1 3PU, United Kingdom \\ ${ }^{2}$ The Hamburg Centre for Ultrafast Imaging, Luruper Chaussee 149, D-22761 Hamburg, Germany \\ ${ }^{3}$ Max Planck Institute for the Structure and Dynamics of Matter, Luruper Chaussee 149, D-22761 Hamburg, Germany
}

(Received 9 November 2020; revised 13 January 2021; accepted 19 January 2021; published 28 January 2021)

\begin{abstract}
Under the action of coherent periodic driving a generic quantum system will undergo Floquet heating and continuously absorb energy until it reaches a featureless thermal state. The phase-space constraints induced by certain symmetries can, however, prevent this and allow the system to dynamically form robust steady states with off-diagonal long-range order. In this work, we take the Hubbard model on an arbitrary lattice with arbitrary filling and, by simultaneously diagonalizing the two possible SU(2) symmetries of the system, we analytically construct the correlated steady states for different symmetry classes of driving. This construction allows us to make verifiable, quantitative predictions about the long-range particle-hole and spin-exchange correlations that these states can possess. In the case when both SU(2) symmetries are preserved in the thermodynamic limit we show how the driving can be used to form a unique condensate which simultaneously hosts particle-hole and spin-wave order.
\end{abstract}

DOI: 10.1103/PhysRevB.103.035146

\section{INTRODUCTION}

Coherent driving has established itself as a fundamental tool for controlling and manipulating the states of quantum systems, from implementing high fidelity gates in few-qubit systems [1] to inducing phase transitions in many-body optical lattices [2]. Within this paradigm, recent experiments have observed how intense laser pulses in the midinfrared regime can transiently induce superconducting features - such as the opening of a gap in the real part of the optical conductivity and vanishing resistivity - when driving various solid state materials out of equilibrium [3-11].

In order to understand seminal results such as these-and more generally the role coherent driving plays in altering the microscopic properties of many-body systems - significant theoretical studies have been undertaken. Floquet theory can be used to understand how periodic driving can modify the parameters of the system and create additional terms on top of the undriven Hamiltonian. This renormalization results in an effective Hamiltonian which on transient scales can, for example, favor superconducting prethermal states [12-25], suppress wave-packet spreading and induce dynamical localization in a many-body bosonic gas [26], control spin-charge separation in a fermionic system [27], or stabilize exotic spinliquid states in frustrated systems [28].

It is inevitable, however, that due to Floquet heating a generic periodically driven quantum system will continuously absorb energy from the driving field. This heating competes with any transient order established by the effective Hamiltonian, causing it to melt away and leading to the formation of a featureless, infinite temperature state in the long-time

\footnotetext{
*joseph.tindall@physics.ox.ac.uk
}

limit $[29,30]$. As a result, engineering Floquet Hamiltonians which are stable to heating on long timescales, allowing their prethermal states to be transiently observable is a current research endeavor attracting significant attention [31-38].

In contrast to these efforts to mitigate the effects of heating in driven systems, recent theoretical work has shown how the presence of SU(2) symmetries in the fermionic Hubbard model can prevent featureless thermalization and result in the formation of correlated, ordered states as the system heats up $[39,40] —$ a mechanism termed 'heating-induced order.' The phase space constraints induced by these symmetries mean the system is forced to relax towards steady states with off-diagonal long-range order as it absorbs energy from an external source. Currently, however, this has only been demonstrated numerically for the case when a single SU(2) symmetry is preserved in small, finite-size instances of the half-filled Hubbard chain [40].

In this work we go beyond this, taking the driven Hubbard model on an arbitrary graph at arbitrary filling and analytically constructing the correlated steady states. We achieve this construction by simultaneously diagonalizing the irreducible representation of the two possible SU(2) symmetries of the system, and through it we can analytically calculate the steady state spin-exchange and particle-hole correlations, at any distance. We verify our analytical results with exact diagonalization calculations and analyze how the long-time correlations depend on factors such as the filling, graph size, initial state, and, crucially, the symmetries the driving possesses. Moreover, we provide the necessary conditions for the steady state correlations to remain finite in the thermodynamic limit. This leads us to show how, in cases where both $\mathrm{SU}(2)$ symmetries are preserved, the driving can be used to merge two independent condensates and create a unique spin- $\eta$ condensate which hosts both spin-exchange and particle-hole 
off-diagonal long-range order. Finally, we discuss possible experimental setups of the driven Hubbard model where the requisite symmetries are preserved in order to observe the formation of such unique, correlated states.

\section{THEORY}

As a starting point we consider the long-time states of a quantum system, with Hamiltonian $H$, subject to continued periodic driving under the modified Hamiltonian $H+H_{D}(t)$, where $H_{D}(t)$ is the time-dependent periodic driving term $H_{D}(t+T)=H_{D}(t)$. We then assume that we can find a set of $X$ operators $C=\left\{C_{1}, C_{2}, \ldots, C_{X}\right\}$, which form a linearly independent, irreducible representation of the symmetries of the system satisfying $\left[C_{i}, C_{j}\right]=0 \forall i, j$. We note that while this is not always possible in a general quantum system, for the cases we consider in this work we are able to identify the completely commuting set $C$. Being a representation of the symmetries of the system we also clearly have that each member of $C$ satisfies

$$
\left[H+H_{D}(t), C_{i}\right]=0, \quad i=1, \ldots, X .
$$

Under the action of $H+H_{D}(t)$ the system will continuously absorb energy until, in the long-time limit, it reaches a state of maximum entropy [29]. The system is, however, under the constraint that its probability distribution over the eigenspace of the operators in $C$ must always be conserved. Consequently, the long-time state of the system will effectively have the form ${ }^{1}$

$$
\lim _{t \rightarrow \infty} \rho(t)=\rho_{\infty}=\sum_{\alpha=\left(\alpha_{1}, \alpha_{2}, \ldots \alpha_{N}\right)} P_{\alpha} \sum_{\beta=1}^{D_{\alpha}} \alpha, \beta \alpha, \beta,
$$

with $\sum_{\alpha} P_{\alpha} D_{\alpha}=1$ and the multi-index/quantum number $\alpha$ running over the combinations of possible eigenvalues of the operators in $C$. For a given $\alpha$, the $D_{\alpha}$ vectors $\alpha, \beta$ form the basis which simultaneously diagonalizes the operators $C_{1}, C_{2}, \ldots, C_{X}$, and $P_{\alpha}$ is the probability of finding the state in this subspace. This probability must be preserved throughout the dynamics and thus

$$
P_{\alpha}=\sum_{\beta=1}^{D_{\alpha}} \operatorname{Tr}(\rho(0) \alpha, \beta \alpha, \beta),
$$

where $\rho(0)$ is the initial state of the system. If the complete basis $\{\alpha, \beta\}$ can be constructed and the corresponding probabilities $P_{\alpha}$ calculated, then the long-time state of the system is known.

The structure and properties of the set $C$ has a significant influence on the properties of the steady state $\rho_{\infty}$. For example, consider the case of a many-body lattice with a single $\mathrm{U}(1)$ symmetry such as the total particle number. The corresponding total number operator is diagonal in the Fock

\footnotetext{
${ }^{1}$ While clearly in a closed system a pure state will always remain pure, this mixed state ansatz is reasonable in a many-body system as the energy of the driving will have scrambled the phases of the wave function sufficiently to destroy any coherences between the $\alpha, \beta$ eigenstates [30].
}

basis and thus, by Eq. (2), so is $\rho_{\infty}$. All the states in the Fock basis can be written as a product state over the different lattice sites, making $\rho_{\infty}$ a featureless (outside of the well-defined particle number), unentangled thermal state.

The same cannot be said, however, for more complicated symmetries. In the case of an SU(n) symmetry for a many-body lattice, one can form the set $C$ by using the generators to construct the $n-1$ Casimir operators which are fully independent of the lattice structure and thus completely translationally symmetric. The basis which simultaneously diagonalizes these Casimir operators is the basis in which $\rho_{\infty}$ is diagonal. These basis states cannot all be written as product states over the different lattice sites and often contain correlations in the form of excitations which are spread between sites. Through Eq. (2) the long-time state will inherit these properties, along with the complete translational invariance of the Casimir operators, and thus possess correlations or excitations which are independent of the lattice geometry, i.e., they are completely uniform with distance. This induction of uniform, long-range correlations by heating an SU(n) symmetric system up to its steady state has been termed 'heating-induced order' and has been studied numerically on small, finite-sized half-filled Hubbard lattices with a single preserved SU(2) symmetry $[39,40]$.

We note that the formation of these off-diagonal long-range ordered states as $t \rightarrow \infty$ does not exclude the possibility that, due to the driving, one could also observe the emergence of some transient, dynamical order on an intermediate timescale. This would then be followed by the melting of this order and the onset of our robust, steady state off-diagonal long-range order (ODLRO) once sufficient heating has occurred. The formation of transient, dynamical order is, however, usually reliant on a careful choice of driving terms and parameters [14,16,18,19,22-24]. Meanwhile, if the requisite symmetries are satisfied, the emergence of steady state ODLRO is guaranteed due to the inevitability of Floquet heating under periodic driving - giving us significant freedom in the driving fields which can be used to observe heating-induced order.

\section{A. The Hubbard model on an arbitrary graph}

Here, we consider the Hubbard model on an arbitrary graph with arbitrary filling and, by simultaneously diagonalizing the dual SU(2) symmetries, analytically construct the long-time states for the different symmetry classes of driving. We define this graph as $\mathcal{G}=(V, E)$ where $V$ are the vertices (or sites) and $E$ the edges. The Hamiltonian can then be written as

$$
H=-\tau \sum_{V, V^{\prime}} \sum_{\sigma=\uparrow, \downarrow}\left(c_{\sigma, V}^{\dagger} c_{\sigma, V^{\prime}}+\text { H.c. }\right)+U \sum_{V} n_{\uparrow, V} n_{\downarrow, V},
$$

where $c_{\sigma, V}^{\dagger}$ and its adjoint are the usual creation and annihilation operators for a fermion of spin $\sigma$ on vertex $V$. The first summation runs over all the edges in the graph, kinetically coupling together the two sites $V, V^{\prime}$ residing at the ends of each edge with strength $\tau$. The second summation runs over all the vertices in the graph and creates an energy penalty $U$ for vertices simultaneously occupied by both spin species. Additionally, $n_{\sigma, V}$ is the number operator for a particle of spin $\sigma$ on site $V$, and we use the integer $M$ to denote the number of vertices on the graph. We also depict this Hamiltonian in 


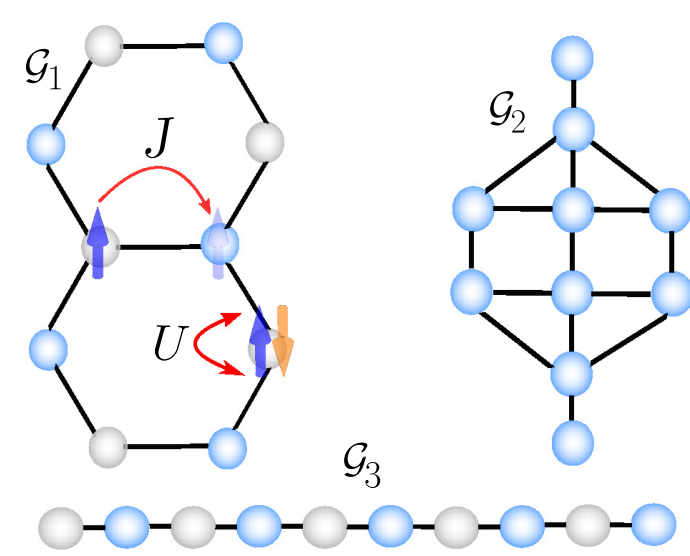

FIG. 1. Fermionic Hubbard model on a graph $\mathcal{G}=\mathcal{G}(V, E)$ where $V$ are the vertices and $E$ are the edges. The $M$ vertices form the lattice sites on which the fermions reside and interact with strength $U$ while the edges form the nearest neighbor bonds over which the fermions can hop with constant amplitude $\tau$. The Hamiltonian is defined in Eq. (4) and $\mathcal{G}_{1}, \mathcal{G}_{2}$, and $\mathcal{G}_{3}$ are the three different $M=10$ vertex graphs we use for our numerics. The gray vs blue sites represent a bipartite splitting on the graphs $\mathcal{G}_{1}$ and $\mathcal{G}_{3}$.

Fig. 1, showing the three finite-size graphs we use for our numerical results, which serve to benchmark our analytical predictions. These analytical predictions, however, can be immediately applied to any graph of any number of vertices.

The Hamiltonian in Eq. (4) has a rich symmetry structure comprised of either one or two SU(2) symmetries [41], which are fundamental to our results. The first, permanent, $\mathrm{SU}(2)$ symmetry can be introduced through the spin-raising operator $S^{+}=\sum_{V} c_{V, \uparrow}^{\dagger} c_{V, \downarrow}$, its conjugate $S^{-}$, and the total magnetization $S^{z}=\sum_{V} n_{\uparrow, V}-n_{\downarrow, V}$. These operators are conserved over all graphs, i.e., $\left[H, S^{ \pm, z}\right]=0, \forall \mathcal{G}$ and we refer to this symmetry as the 'spin' symmetry with the corresponding operators only acting nontrivially on the sites of the lattice occupied by a single fermion (singlons).

The second of the $\mathrm{SU}(2)$ symmetries is introduced through the $\eta$-raising operator $\eta^{+}=\sum_{V} f(V) c_{V, \uparrow}^{\dagger} c_{V, \downarrow}^{\dagger}$, its conjugate $\eta^{-}$, and the modified total number operator $\eta^{z}=\sum_{V}\left(n_{\uparrow, V}+\right.$ $\left.n_{\downarrow, V}-1\right)$. If the graph $\mathcal{G}$ is bipartite, i.e., the vertices can be split into two sets with the edges of the graph only forming connections between the two sets, then $\left[H, \eta^{+} \eta^{-}\right]=0$ if we set the function $f(V)$ to take the values \pm 1 depending on whether the corresponding vertex is in the first or second set. Moreover, we always have $\left[H, \eta^{z}\right]=0 \forall \mathcal{G}$ and thus the $\eta$ operators form an $\mathrm{SU}(2)$ or $\mathrm{U}(1)$ symmetry depending on whether the graph is bipartite or not. The corresponding operators only act nontrivially on the empty and full sites within the lattice and these sites are often referred to as occupied by a 'hole' or a 'doublon' quasiparticle, respectively.

In addition to this we note that there may be additional symmetries that $H$ possesses which will reflect the polygon symmetries of the graph $\mathcal{G}$. For example on an open boundary 1D chain the Hubbard model has a reflection symmetry about the central site, while on more complex geometries there may be multiple reflection and translational shift symmetries [41].

Our goal is to determine the long-time states reached under continued periodic driving on top of the Hamiltonian in
Eq. (4). For simplicity, we will assume that the driving either breaks any polygon symmetries in the system or that they can be ignored due to their sufficiently small effect on the longtime properties of the system. We will see this is a reasonable assumption for the graphs we use and therefore the relevant symmetries to consider in our system are the two possible $\mathrm{SU}(2)$ symmetries of the lattice.

From here on we will also, without loss of generality, fix the quantities $N_{\uparrow}$ and $N_{\downarrow}$ which correspond to the total number of fermions of spin $\uparrow$ and spin $\downarrow$, respectively. Our results in the full Hilbert space can be recovered by performing a direct sum over all possible values of $N_{\uparrow}$ and $N_{\downarrow}$. To keep the equations we derive more concise, we will restrict ourselves to lattices with an even number of sites $M$ and an even total number of particles $N=N_{\uparrow}+N_{\downarrow}$. For brevity we will also introduce the integer quantities $\alpha=(M-N) / 2$ and $\beta=\left(N_{\uparrow}-N_{\downarrow}\right) / 2$.

\section{B. Simultaneously diagonalizing the Hubbard SU(2) Casimir operators}

In order to construct the long-time state for arbitrary driving we must be able to diagonalize the irreducible representation of the dual SU(2) symmetries. This representation, i.e., our set $C$, consists of the two $\mathrm{SU}(2)$ Casimir operators $\eta^{2}=\eta^{+} \eta^{-}+\eta^{-} \eta^{+}+\left(\eta^{z}\right)^{2}$ and $S^{2}=S^{+} S^{-}+S^{-} S^{+}+\left(S^{z}\right)^{2}$ and their corresponding ' $z$ ' operators $\eta^{z}$ and $S^{z}$. By fixing the particle numbers we have already removed the dependency on $\eta^{z}$ and $S^{z}$ and this restriction also means that the eigenvectors of the operator $O^{+} O^{-}$are also those of $O^{-} O^{+}$, where $O$ is either $\eta$ or $S$. Hence, our problem is immediately simplified to simultaneously diagonalizing $S^{+} S^{-}$and $\eta^{+} \eta^{-}$.

We can make progress with this problem by noticing that both operators commute with the total doublon operator $N_{D}=$ $\sum_{V} n_{\uparrow} n_{\downarrow}$. We can therefore simultaneously reduce them into block matrices with the blocks indexed by $i$, the number of doublons on the graph, which ranges from $\operatorname{Max}(0,-2 \alpha)$ to $\operatorname{Min}\left(N_{\uparrow}, N_{\downarrow}\right)$. For a given value of $i$, we observe that there must be $M+i-N$ holes in the graph and so the remaining vertices, or sites, will be occupied by $N_{\uparrow}-i$ and $N_{\downarrow}-i$ singlons of spin $\uparrow$ and $\downarrow$, respectively.

We can use this knowledge to take any given block and arrange the sites of the lattice into two sets, with the first set $(A)$ containing $M+2 i-N$ sites and the second set $(B)$ containing the remaining $N-2 i$ sites. There are $\left(\begin{array}{c}M \\ N-2 i\end{array}\right)$ different ways in which the sites can be arranged in this manner and in $A$ we place all of the doublons and holes while in $B$ we place all of the singlons. If the operator $S_{V}^{+} S_{V^{\prime}}^{-}\left(\eta_{V}^{+} \eta_{V^{\prime}}^{-}\right)$acts on a vertex which is in set $A(B)$ then it will immediately annihilate any given basis state, and thus we can let

$$
\begin{aligned}
& \eta^{+} \eta^{-} \rightarrow\left(\eta^{+} \eta^{-}\right)^{\prime}=\sum_{V, V^{\prime} \in A} \eta_{V}^{+} \eta_{V^{\prime}}^{-}, \\
& S^{+} S^{-} \rightarrow\left(S^{+} S^{-}\right)^{\prime}=\sum_{V, V^{\prime} \in B} S_{V}^{+} S_{V^{\prime}}^{-},
\end{aligned}
$$

and ignore the other terms in these two operators. If we can now construct a state $\eta$, within $A$, which is an eigenvector of $\left(\eta^{+} \eta^{-}\right)^{\prime}$ with eigenvalue $\lambda_{A}$ and a state $S$ within $B$ which is an eigenvector of $\left(S^{+} S^{-}\right)^{\prime}$ with eigenvalue $\lambda_{B}$ then their 'tensor 
TABLE I. Form of the probabilities $P_{k, m}$ which characterize the steady state of the driven Hubbard model [see Eq. (9)]. The probabilities are defined by their relationship to the initial state $\rho(0)$ and the projectors $\mathcal{P}_{k}^{\eta}, \mathcal{P}_{m}^{S}$, and $\mathcal{P}_{m, k}^{S, \eta}$, which are defined in Eq. (10). This relationship changes depending on which of the two SU(2) symmetries are present during the systems evolution to the steady state.

\begin{tabular}{lcr}
\hline \hline Spin Symmetry $\eta$ Symmetry & No & Yes \\
\hline No & $P_{m, k}=$ const. & $P_{m, k}=P_{m} P_{k}, P_{k}=\operatorname{Tr}\left(\rho(0) \mathcal{P}_{k}^{\eta}\right) P_{m}=$ const. \\
Yes & $P_{m, k}=P_{m} P_{k}, P_{k}=$ const., $P_{m}=\operatorname{Tr}\left(\rho(0) \mathcal{P}_{m}^{S}\right)$ & $\operatorname{Tr}\left(\rho(0) \mathcal{P}_{m, k}^{S, \eta}\right)$ \\
\hline \hline
\end{tabular}

product' $\eta \tilde{\otimes} S$ will simultaneously be an eigenvector of both $\eta^{+} \eta^{-}$and $S^{+} S^{-}$on the full graph with eigenvalues $\lambda_{A}$ and $\lambda_{B}$, respectively. The tilde on the tensor product means we will take into account the way the sets $A$ and $B$ were formed and reorder the vertices of the graph back to their original order.

We now need to determine the eigenspectrum of $\left(\eta^{+} \eta^{-}\right)^{\prime}$ and $\left(S^{+} S^{-}\right)^{\prime}$. Crucially, we know that $\left(\eta^{+} \eta^{-}\right)^{\prime}$ and $\left(S^{+} S^{-}\right)^{\prime}$ correspond to the SU(2) Casimir operators in a, respectively, $M+2 i-N$ and $N-2 i$-fold tensor product representation of the fundamental representation of SU(2) $[42,43]$. By exploiting the 'ladder' structure of $S U(2)$ representations we can determine the eigenvalues of $\left(\eta^{+} \eta^{-}\right)^{\prime}$ and $\left(S^{+} S^{-}\right)^{\prime}$ as

$$
\begin{aligned}
& \lambda_{\eta}(k)=k(k+1)-\alpha(\alpha+1), \quad k=|\alpha|, \ldots \alpha+i \\
& \lambda_{s}(m)=m(m+1)-\beta(\beta+1), \quad m=|\beta|, \ldots, N / 2-i .
\end{aligned}
$$

These eigenvalues have the following degeneracies

$$
\begin{aligned}
& D_{\eta}(k)=C\left(\alpha+i+k, \alpha+i-k-\delta_{k}\right), \\
& D_{S}(m)=C\left(N / 2-i+m, N / 2-i-m-\delta_{m}\right),
\end{aligned}
$$

where $\delta_{a}$ is the Kronecker delta function and $C(x, y) \equiv C_{y}^{x}$ is the Catalan triangle number [44]

$$
C_{y}^{x}= \begin{cases}\frac{(x+y) !(x-y+1)}{y !(x+1) !} & x, y>0 \\ 1 & \text { otherwise. }\end{cases}
$$

With this knowledge we can denote $\eta_{k, l}$ and $S_{m, n}$ as the eigenvectors of $\left(\eta^{+} \eta^{-}\right)^{\prime}$ and $\left(S^{+} S^{-}\right)^{\prime}$ where $k$ and $m$ index the respective eigenvalues and $l$ and $n$ run through the degenerate eigenvectors for a given $k$ and $m$. The state $\psi=\eta_{k, l} \tilde{\otimes} S_{m, n}$ is then an eigenvector of $\eta^{+} \eta^{-}$and $S^{+} S^{-}$on the full graph $\mathcal{G}$. The relevant indices to specify the state $\psi$ are then $\psi_{i, j, k, l, m, n}$ where $i$ is the number of doublons, $j$ indexes the $\left(\begin{array}{c}L \\ N-2 i\end{array}\right)$ ways in which the lattice can be split into the two aforementioned sets, and $k$ and $l$ index the degenerate eigenvectors of $\left(\eta^{+} \eta^{-}\right)^{\prime}$ while $m$ and $n$ do the same for $\left(S^{+} S^{-}\right)^{\prime}$. The states $\psi$ form a complete, orthonormal basis which diagonalize $\eta^{+} \eta^{-}$and $S^{+} S^{-}$for the given filling.

\section{Steady states of the driven Hubbard model}

We can now combine the basis we have constructed with the general result of Eq. (2) and write down the steady state $\rho_{\infty}$ of the periodically driven Hubbard model on an arbitrary graph with arbitrary filling as

$$
\rho_{\infty}=\frac{1}{Z} \sum_{i=\operatorname{Max}(0,-2 \alpha)}^{\operatorname{Min}\left(N_{\uparrow}, N_{\downarrow}\right)} \sum_{k=|\alpha|}^{\alpha+i} \sum_{m=|\beta|}^{N / 2-i} P_{k, m} \sum_{l=1}^{C_{\alpha+i-k-\delta_{k}}^{\alpha+i+k}} \sum_{n=1}^{C_{N / 2-i-m-\delta_{m}}^{N / 2-i+m}} \sum_{j=1}^{\left(\begin{array}{c}
{ }^{L} \\
N-2 i
\end{array}\right)} \psi_{i, j, k, l, m, n} \psi_{i, j, k, l, m, n},
$$

where $Z$ is the partition function and the values $P_{k, m}$ are a series of probabilities [analogous to the $P_{\alpha}$ in Eq. (2)] which are dependent on both the initial state as well as which, if any, of the SU(2) symmetries are present in the system. This dependency is encapsulated by Table I, where we have also introduced the following projectors

$$
\begin{aligned}
\mathcal{P}_{k}^{\eta} & =\sum_{i, j, m, n, l} \psi_{i, j, k, l, m, n} \psi_{i, j, k, l, m, n}, \\
\mathcal{P}_{m}^{S} & =\sum_{i, j, k, n, l} \psi_{i, j, k, l, m, n} \psi_{i, j, k, l, m, n}, \\
\mathcal{P}_{m, k}^{S, \eta} & =\sum_{i, j, n, l} \psi_{i, j, k, l, m, n} \psi_{i, j, k, l, m, n},
\end{aligned}
$$

with the indices used all retaining their original meaning and ranges from Eq. (9).

Table I, alongside Eq. (9), allows us to classify and write down the steady states of the driven Hubbard model on an arbitrary graph. This classification is based on which of the
SU(2) symmetries are present during the time evolution anddue to the excited, entangled nature of the eigenstates of the SU(2) Casimir operators-the long-time state will contain long-range correlations in the channels corresponding to the preserved symmetries. Meanwhile, in the channels where the underlying symmetry was not present the constant nature of the probabilities means all excitations are equally likely and they will destructively interfere with each other to ensure there is no long-range order in that channel.

Given the probabilities $P_{k, m}$ we can calculate a number of properties of the state in Eq. (9). For example we can immediately deduce the moments of the doublon number $N_{d}$

$$
\begin{aligned}
\left\langle N_{d}^{\alpha}\right\rangle & =\frac{1}{Z} \sum_{i} i^{\alpha} f(i), \\
f(i) & =\sum_{k, m} P_{k, m} C_{\alpha+i-k-\delta_{k}}^{\alpha+i+k} C_{N / 2-i-m-\delta_{m}}^{N / 2-i+m}\left(\begin{array}{c}
M \\
N-2 i
\end{array}\right) .
\end{aligned}
$$


These equations are useful because we can take advantage of the distance invariance of correlations in the long-time state and use the first moment of the doublon number $(\alpha=1)$, along with the initial values $\left\langle\eta^{+} \eta^{-}\right\rangle$and $\left\langle S^{+} S^{-}\right\rangle$, to directly extract values for the off-diagonal spin-exchange and particlehole order parameters $\left\langle S_{V}^{+} S_{V^{\prime}}^{-}\right\rangle$and $\left\langle\eta_{V}^{+} \eta_{V^{\prime}}^{-}\right\rangle$. Specifically, we know that

$$
\left\langle\eta^{+} \eta^{-}\right\rangle=\left\langle N_{d}^{1}\right\rangle+M(M-1)\left\langle\eta_{V}^{+} \eta_{V^{\prime}}^{-}\right\rangle,
$$

and

$$
\left\langle S^{+} S^{-}\right\rangle=N_{\uparrow}-\left\langle N_{d}^{1}\right\rangle+M(M-1)\left\langle S_{V}^{+} S_{V^{\prime}}^{-}\right\rangle,
$$

where $V \neq V^{\prime}$. Moreover, higher moments of the doublon number $\alpha>1$ provide access to multipoint correlators in the $\eta$ and spin symmetry sectors (for example, we can show that $\left.\left\langle n_{\uparrow, V} n_{\downarrow, V} n_{\uparrow, V^{\prime}} n_{\downarrow, V^{\prime}}\right\rangle \propto\left\langle N_{d}^{2}\right\rangle-\left\langle N_{d}^{1}\right\rangle\right)$.

In principle, however, in order to calculate the moments of the doublon number we need to know the exact values of the $P_{k, m}$ s which, in some cases, could be quite complicated and would involve taking a number of projective measurements on the initial state. We find from our equations, however, that the first moment of the doublon number is only dependent on the probabilities $P_{k, m}$ through its relationship to $\left\langle\eta^{+} \eta^{-}\right\rangle$and $\left\langle S^{+} S^{-}\right\rangle$and thus knowledge of these two values, and the graph size and filling, is enough to calculate $\left\langle N_{d}^{1}\right\rangle$. The steady state off-diagonal order parameters $\left\langle\eta_{V}^{+} \eta_{V^{\prime}}^{-}\right\rangle$and $\left\langle S_{V}^{+} S_{V^{\prime}}^{-}\right\rangle$then follow immediately from Eq. (12) and the corresponding 'spin' version.

These quantities are particularly important because when finite in the thermodynamic limit and completely uniform with distance, the latter of which is automatically satisfied by $\rho_{\infty}$, they describe the existence of a spin-wave or $\eta$ condensate. These condensates are underpinned by excitations which are completely spread out in space and in an $\eta$ condensate the long-range finite value of $\left\langle\eta_{V}^{+} \eta_{V^{\prime}}^{-}\right\rangle$directly implies superconductivity as the Meissner effect and flux quantization can be observed $[45,46]$.

\section{RESULTS: HUBBARD MODEL UNDER GENERIC DRIVING}

We now demonstrate the results we have derived explicitly, first focusing on the case where a single $\mathrm{SU}(2)$ symmetry is preserved and then moving on to the case where both $\mathrm{SU}(2)$ symmetries are preserved. We note that the scenario where both symmetries are not preserved needs no attention as it is trivial and the long-time state will simply be a featureless thermal state with a fixed particle number.

\section{A. Single symmetry preservation}

We consider a setup in which the spin SU(2) symmetry is present while the $\eta \mathrm{SU}(2)$ symmetry is not. These results are immediately analogous to the case where the spin symmetry is not present and the $\eta$ symmetry is, which was studied in Ref. [40] but only for a small, half-filled chain. We introduce the graph measure $d\left(V, V^{\prime}\right)$ which is the minimum number of edges that must be traversed to move between the vertices $V$ and $V^{\prime}$. With this measure we can then define the correlation
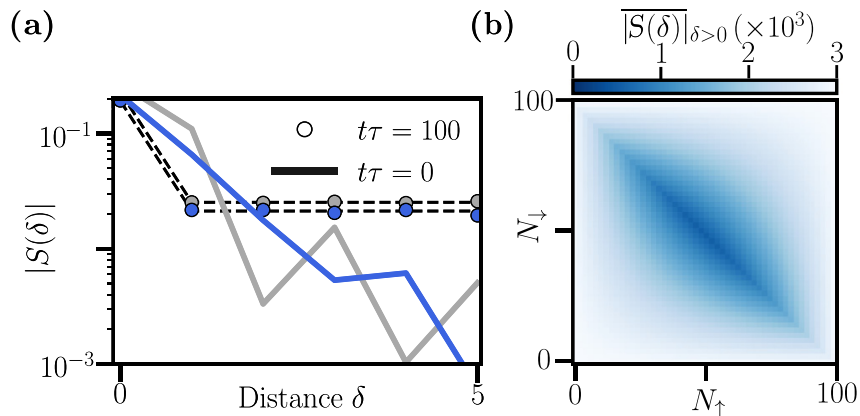

FIG. 2. (a) Spin-exchange correlations versus distance $\delta$, see Eq. (14), for the periodically driven graph $\mathcal{G}_{1}$ pictured in Fig. 1, with the site index $V$ running from 1 to 10 , starting at $V=1$ for the bottom left site and increasing in an anticlockwise manner. The driving term we use is of the form $H_{D}=A \cos (\Omega t) \sum_{V} V n_{V}$ and the gray vs blue markers/lines correspond to the fillings $N_{\uparrow}=N_{\downarrow}=5$ and $N_{\uparrow}=N_{\downarrow}=3$, respectively. At time $t \tau=0$ the system is initialized in the ground state of the undriven Hamiltonian $H$ with $U=0.5 \tau$ and then evolved under the Hamiltonian $H+H_{D}$ with $U=\tau, A=6.0 \tau$, and $\Omega=1.0 \tau$. The solid lines vs markers indicate the correlations at $t \tau=0$ and $t \tau=100$, respectively. The black-dotted lines give the exact results in the long-time limit. (b) Off-diagonal spin-exchange order as a function of the numbers of $\uparrow$ and $\downarrow$ fermions in the steady state of a driven, arbitrary, $M=100$ vertex Hubbard graph where the spin $\mathrm{SU}(2)$ symmetry is preserved while the $\eta \mathrm{SU}(2)$ symmetry is not. The system is initialized with $\left\langle S^{+} S^{-}\right\rangle=0$.

function

$$
O(\delta)=\frac{1}{\mathcal{N}} \sum_{\substack{\left\langle V, V^{\prime}\right\rangle \\ d\left(V, V^{\prime}\right)=\delta}}\left\langle O_{V}^{+} O_{V^{\prime}}^{-}\right\rangle,
$$

where $O$ is either $S$ or $\eta$, the summation is over all pairs of vertices where $d\left(V, V^{\prime}\right)=\delta$, and $\mathcal{N}$ is the number of pairs of vertices which satisfy $d\left(V, V^{\prime}\right)=\delta$. Hence, $O(\delta)$ measures the average of the spin-exchange or particle-hole correlations at a distance $\delta$ for any given graph, and we can introduce $\overline{|O(\delta)|_{\delta>l}}$ as the average magnitude of these correlations at distances greater than $l$.

In Fig. 2(a) we demonstrate agreement, for two different particle fillings, between the equations in the previous section and exact diagonalization code which reaches the long-time limit of the graph $\mathcal{G}_{1}$ from Fig. 1. As the driving explicitly breaks $\eta$ symmetry the long-time prediction from these equations is independent of the lattice structure and whether it is bipartite or not. Figure 2 shows that when driving the ground state out of equilibrium, the preservation of $\left\langle S^{+} S^{-}\right\rangle$ under driving causes the establishment of completely uniform, long-range spin-wave order in the long-time limit, with a significant enhancement of the long-range correlations. This long-range order is largest at the higher filling and, more generally, our equations show that the steady state order will always be maximized when the system is closest to zero total magnetization and half filling, where the largest number of spin-exchange excitations are available. In Fig. 2(b) we show this result explicitly, observing significant order around this half-filled nonmagnetic point which then decays to zero as either of the filling numbers, $N_{\uparrow}$ and $N_{\downarrow}$, approach their 

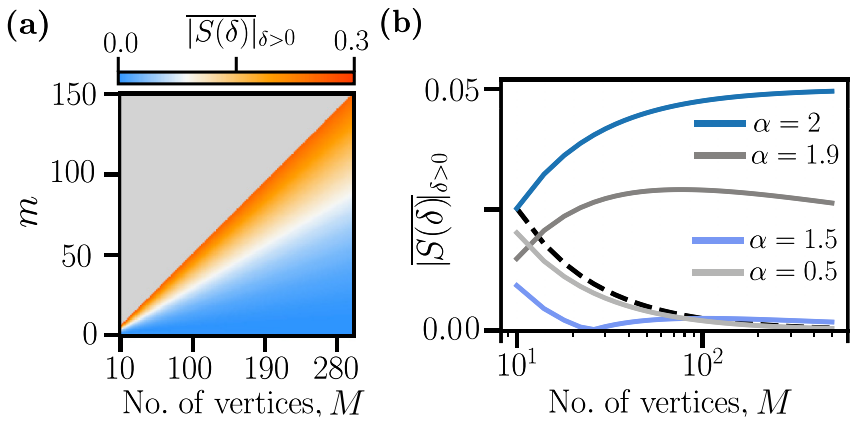

FIG. 3. Scaling of the steady state off-diagonal spin-exchange correlations with the number of vertices and initial value of $\left\langle S^{+} S^{-}\right\rangle=\operatorname{Tr}\left(\rho(0) S^{+} S^{-}\right)$for the half-filled Hubbard model on an arbitrary graph. The system is time evolved under driving which preserves the spin $\mathrm{SU}(2)$ symmetry and breaks the $\eta$ symmetry. (a) Scaling versus both system size and $m$ where $m=(-1+$ $\left.\sqrt{1+4\left\langle S^{+} S^{-}\right\rangle}\right) / 2$. (b) Scaling with system size for various initial states. The solid lines correspond to the initial values $\left\langle S^{+} S^{-}\right\rangle=$ $M^{\alpha} / 20$ for varying $\alpha$ while the dashed line is for $\left\langle S^{+} S^{-}\right\rangle=0$.

maximal or minimal values (where it is not possible to perform a spin-exchange $S_{V}^{+} S_{V^{\prime}}^{-}$without annihilating the state of the system).

We then concentrate on the nonmagnetic half-filled point, where the order is maximized, and in Fig. 3 plot the magnitude of the long-time spin-exchange order for a large range of system sizes and initial values $\operatorname{Tr}\left(\rho(0) S^{+} S^{-}\right)$. These results apply to any driven Hubbard graph where the spin SU(2) symmetry is preserved and the $\eta$ symmetry is not, which could be a result of the driving or the underlying lattice structure.

Notably, as system size increases there is a growing space of initial values where there is significant spin-wave order in the long-time limit. In fact we can easily argue that the spin-wave order will be finite in the thermodynamic limit of any graph if, and only if, the initial state satisfies $\left\langle S^{+} S^{-}\right\rangle \propto$ $M^{2}$, with Fig. 3(b) showing this explicitly. Such states are already likely to have finite long-range spin correlations to satisfy this requirement, however the driving will still act to renormalize these correlations and make them completely uniform with distance, stabilizing the spin-wave order. Meanwhile, for simulations which start in the ground state (where $\left.\left\langle S^{+} S^{-}\right\rangle \equiv 0\right)$ the spin order asymptotically tends to 0 as $1 / M$ but remains finite for any finite-size system-with the dynamics underpinned by a drastic amplification of the long-range correlations at the expense of the short-range ones.

Interestingly, in Ref. [12], a type of spin preserving periodic driving was studied for the 1D Hubbard chain in the thermodynamic limit. There it was shown how driving the ground state can renormalize the exchange parameters in the system and transiently enhance long-range singlet pairing. Even though the long-time spin order will be 0 , our results here suggest this transient response could be a result of the preservation of $\left\langle S^{+} S^{-}\right\rangle$. Under driving, this preservation forces a drastic reorganization of the spin degrees of freedom, which will involve a transient enhancement of the long-range correlations at the expense of the shorter ones, before they mutually decay away to 0 in the long-time limit.
Despite the ground state of hypercubic Hubbard lattices possessing the smallest possible value of $\left\langle S^{+} S^{-}\right\rangle$, our equations show that the magnitude of the induced off-diagonal spin order $\left|\left\langle S_{V}^{+} S_{V^{\prime}}^{-}\right\rangle\right|$under driving which preserves the $\mathrm{SU}(2)$ spin symmetry is larger than that for any initial states which have finite $\left\langle S^{+} S^{-}\right\rangle<(M-1) / 2$. This is because the steady state spin order $\left\langle S_{V}^{+} S_{V^{\prime}}^{-}\right\rangle$is a monotonic function of $\left\langle S^{+} S^{-}\right\rangle$ but is negative for finite $M$ and $\left\langle S^{+} S^{-}\right\rangle<M / 4$, at which point it changes sign. Hence, the ground state spin order is the most negative and it can be shown from our equations that the magnitude of this order is larger than that of any other states in the range $0<\left\langle S^{+} S^{-}\right\rangle<(M-1) / 2$. On a halffilled hypercubic lattice, this range includes all initial states in thermal equilibrium $\rho(0) \propto e^{-\beta H}$ as the value of $\left\langle S^{+} S^{-}\right\rangle$ for these states monotonically increases from 0 to $M / 4$ as the inverse temperature $\beta$ decreases from $\infty$ to 0 . Hence, for these thermal initial states on a finite sized lattice, the magnitude of the steady state spin order is maximized for the ground state and remains finite for any finite temperature initial state-asymptotically tending to 0 as the temperature of the initial state approaches $\infty$ where $\left\langle S^{+} S^{-}\right\rangle=M / 4$.

Outside of hypercubic lattices it is harder to make statements about the long-time order which will form from an initial state in thermal equilibrium. This is because the relationship between the expectation value of the SU(2) Casimir operator (which determines the amplitude of the long-time order) and $\beta$ is likely to be more complex. This could, however, lead to the exciting possibility of heating $\rho(0) \propto e^{-\beta H}$ under certain symmetries and forming a state with uniform, finite, off-diagonal order even in the thermodynamic limit dynamically transforming a system in thermodynamic equilibrium into a 'hot' condensate.

It is worth emphasizing that in this paper we have taken the hopping strength $\tau$ to be homogeneous across all edges of the lattice. The spin SU(2) symmetry of the Hubbard model is, however, preserved even in the case of an inhomogeneous hopping strength and so our results in this section immediately apply to this more general scenario.

\section{B. Both SU(2) symmetries preserved}

We now move to the case where the driving preserves both $\mathrm{SU}(2)$ symmetries and in order for this preservation to also be true for the full Hamiltonian $H+H_{D}(t)$ we require the underlying graph to be bipartite, which we will assume in our analytical calculations. For our numerics, the graphs $\mathcal{G}_{1}$ and $\mathcal{G}_{3}$ are bipartite while $\mathcal{G}_{2}$ is not, and so we shall see that this leads to distinctly different dynamics when they are driven. We fix ourselves to symmetric half filling $\left(N_{\uparrow}=N_{\downarrow}=M / 2\right)$ where the long-time order will be maximal due to the maximum availability of both particle-hole and spin-exchange excitations. In Figs. 4(a) and 4(b) we start in a thermal state and apply driving which preserves both $\mathrm{SU}(2)$ symmetries to the three different lattice structures $\mathcal{G}_{1}, \mathcal{G}_{2}$, and $\mathcal{G}_{3}$, calculating the average amplitude of the correlations between all pairs of sites not connected by an edge. In the particle-hole channel, we observe a pronounced increase in this average, with the large value of $U$ having suppressed them in the initial thermal state. The average in the spin-exchange sector instead remains relatively constant, but the inset in Fig. 4(c) shows that the 

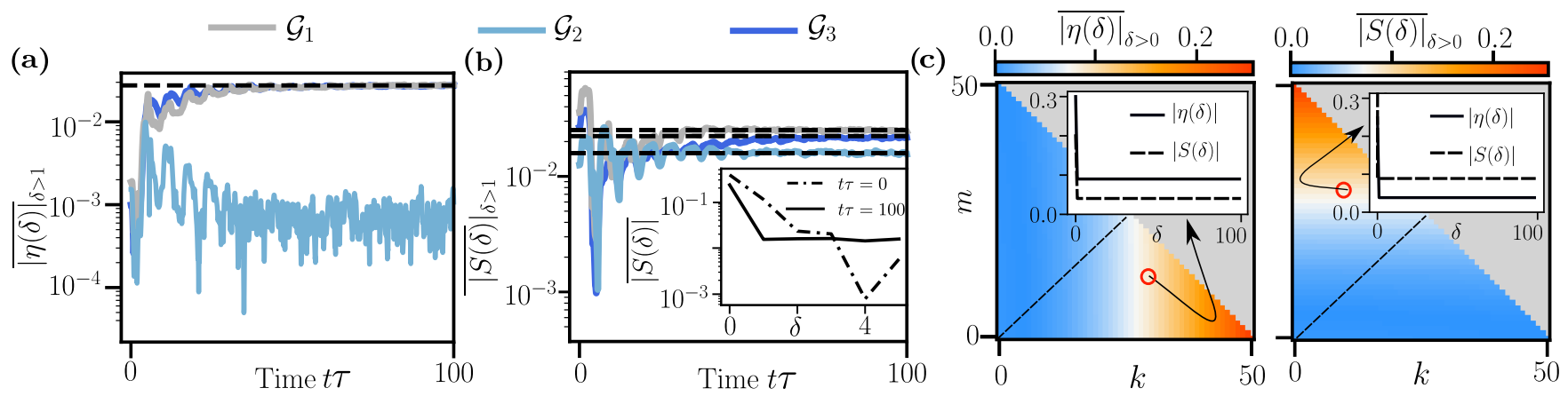

FIG. 4. (a),(b) Off-diagonal spin and $\eta$ correlations versus time and distance for three different half-filled Hubbard lattices with driving of the form $H_{D}=\delta U \cos (\omega t) \sum_{i} n_{\uparrow, i} n_{\downarrow, i}$ and the bare Hamiltonian $H$ as defined in Eq. (4) with $U=4.0 \tau$. The system is initialized, at time $t \tau=0$, in the thermal state $\rho \propto \exp (-\beta H)$ with $\beta \tau=5$ and then time evolved under $H$ with $U=4.0 \tau, \delta U=1.5 \tau$, and $\omega=1.0 \tau$. Black-dotted lines represent the long-time analytical predictions for the three respective graphs. (Inset) Spin correlations versus distance at times $t \tau=0$ and $t \tau=100$ for the lattice $\mathcal{G}_{2}$. (c) Map of the doublon/ spin order of long-time states of the Hubbard model on a 100 vertex bipartite graph with driving which preserves both $\mathrm{SU}(2)$ symmetries. The indices $m$ and $k$ are dependent on the initial values of the spin and $\eta$ symmetry via $m=\left(-1+\sqrt{1+4\left\langle S^{+} S^{-}\right\rangle}\right) / 2$ and $k=\left(-1+\sqrt{1+4\left\langle\eta^{+} \eta^{-}\right\rangle}\right) / 2$. The two maps are related via a reflection over the black-dotted line. (Insets) Doublon (solid line) and spin (dashed line) order for the long-time state at the circled point on the map.

driving has still reordered these correlations to be completely uniform with distance. We find our predictions from Eq. (11) are in perfect quantitative agreement with the long-time order observed in these states and, as we expect, the nonbipartite graph $\mathcal{G}_{2}$ cannot support particle-hole order as it lacks the requisite symmetry.

In Fig. 4(c) we present maps of the long-time off-diagonal particle-hole and spin-exchange order as a function of the $\eta$ and spin eigenvalues of the initial state for a bipartite graph with $M=100$ sites. There is a whole manifold of states with significant, co-existing, particle-hole and spin-exchange order, and we present an example state in the inset of each map. Here, we can also show that, similar to the single symmetry case, when starting in the ground state the off-diagonal spin and $\eta$ order always remains finite for finite systems but asymptotically decays to 0 as $1 / M$.

In fact, in the thermodynamic limit, the condition $\left\langle\eta^{+} \eta^{-}\right\rangle \propto M^{2}$ or $\left\langle S^{+} S^{-}\right\rangle \propto M^{2}$ is necessary to observe finite ODLRO in the $\eta$ and spin sectors, respectively. These two conditions are not mutually exclusive, allowing us to exploit the driving to form a unique spin- $\eta$ condensate. Specifically, consider a bipartite $M$ site lattice which hosts the initial state $\left(S^{+}\right)^{M / 4} \chi_{1} \otimes\left(\eta^{+}\right)^{M / 4} \chi_{2}$, where $\chi_{1}=\downarrow, \downarrow, \ldots, \downarrow$ on $M / 2$ of the sites and $\chi_{2}$ is the vacuum state on the other $M / 2$ sites. This state consists of two independent condensates-a spinwave and particle-hole condensate- each confined to one half of the lattice. Under driving which preserves both SU(2) symmetries, and a Hubbard Hamiltonian $H$ over the full lattice, the dynamics will involve the system heating up while conserving the values of $\left\langle S^{+} S^{-}\right\rangle$and $\left\langle\eta^{+} \eta^{-}\right\rangle$- forcing the condensates to merge and phase lock into a larger, single condensate which, remarkably, hosts ODLRO in the particle-hole and spinexchange sectors simultaneously. Specifically, our equations tell us $\lim _{M \rightarrow \infty} \overline{|\eta(\delta)|}_{\delta>0}=\lim _{M \rightarrow \infty} \overline{|S(\delta)|}_{\delta>0}=0.0625$, i.e., there is completely ODLRO in both symmetry sectors. The interplay between heating induced by the driving and the preservation of $\mathrm{SU}(2)$ symmetries has therefore led to the formation of a unique state of matter which is simultaneously a superconductor and a spin-wave condensate.
In Fig. 5 we picture this process of merging two condensates for a Hubbard chain. The actual lattice structure is not important and such a process could be performed by driving condensates which are initialized on two halves of any bipartite lattice. Moreover, this process could also be used to merge two condensates of the same type, such as states of the form $\left(S^{+}\right)^{M / 4} \chi_{1} \otimes\left(S^{+}\right)^{M / 4} \chi_{1}$ or $\left(\eta^{+}\right)^{M / 4} \chi_{2} \otimes\left(\eta^{+}\right)^{M / 4} \chi_{2}$. In this case the driving only needs to preserve the relevant SU(2) symmetry in order to phase lock and merge the two condensates into a larger one.

\section{Experimental implementation of SU(2) symmetry preservation}

Finally, it is important to discuss how driving which preserves the SU(2) symmetries of the Hubbard model can be achieved experimentally. If we consider an optical lattice implementation of the Hubbard model [47-49] we can take advantage of the fact the Hubbard interaction and hopping strengths have a well defined relationship with the depth and separation of the potential minima which form the optical lattice sites. These quantities can be directly controlled, and made to oscillate, by modulating the standing-wave interference pattern which generates the potential landscape-a process which has already led to the experimental realization of a Hubbard Hamiltonian with time-dependent parameters [50] and could be used to realize the unique states we have observed here, including the exotic $\eta$-spin condensate in Fig. 5.

Moreover, in a quantum materials setting, a recent experiment has shown how laser excitation of the vibrational modes of the organic charge transfer salt $\kappa-(\mathrm{BEDT}-\mathrm{TTF})_{2} \mathrm{Cu}\left[\mathrm{N}(\mathrm{CN})_{2}\right] \mathrm{Br}$, whose conducting layers can be described by a triangular Hubbard model, leads to the formation of transient superconducting features [11]. Density functional theory modeling of the material has shown that the laser excitation induces a periodic time dependence in the parameters of the triangular Hubbard Hamiltonian which, in combination with the irregular geometry of the system, leads to the system transiently establishing particle-hole order 

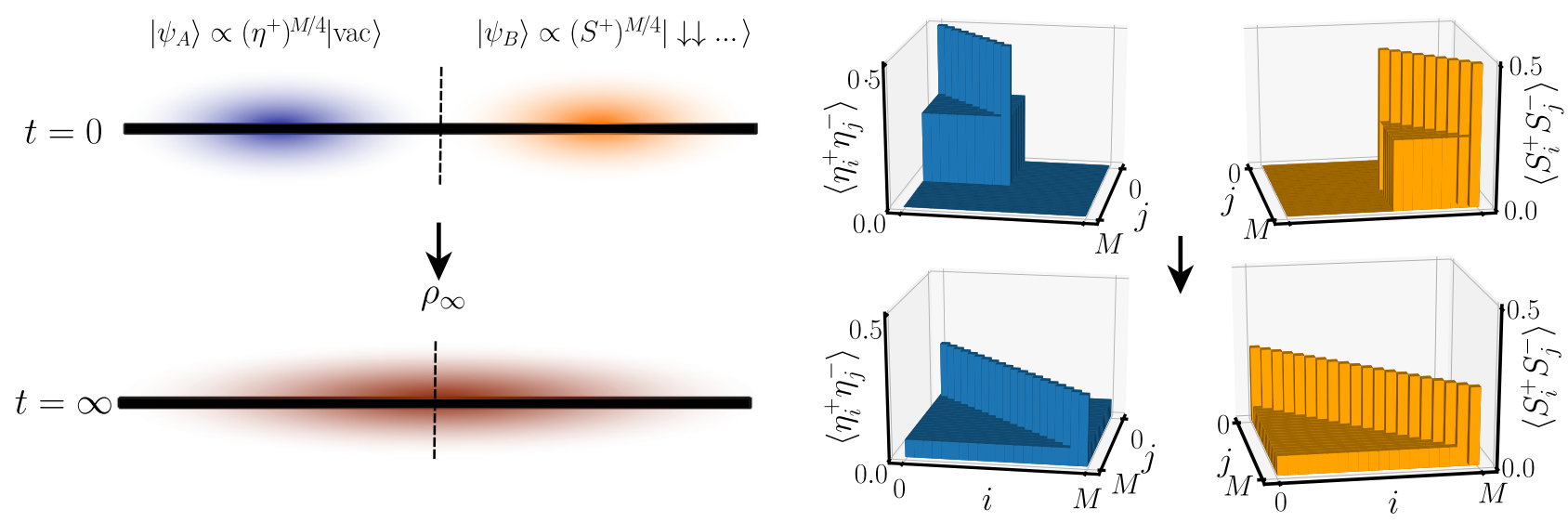

FIG. 5. Merging of two condensates under long-time driving. The Hubbard model on a 1D chain is split into two independent halves which contain an $\eta$ and spin-wave condensate, respectively. The system is then time evolved under generic driving which respects both SU(2) symmetries of the model, causing the condensates to merge into a single hybrid spin- $\eta$ condensate. (Left) Pictorial depiction of the process. (Right) Matrices of $\eta$ and spin-exchange correlations for the whole lattice in the thermodynamic limit $(M \rightarrow \infty)$ at times $t=0$ and $t=\infty$; $i$ and $j$ index the different sites of the chain.

as it absorbs energy from the driving field [51]. The numerical calculations in Ref. [51] thus provide a possible connection between the mechanism of heating-induced order and this recent experiment.

It is also worth mentioning that, in this work, we have considered the situation where the relevant $\mathrm{SU}(2)$ symmetries are completely preserved and so the correlated states we observe will form in the long-time limit and persist indefinitely, i.e., they are not prethermal states but are exact steady states of the system. In a realistic experimental setup, however, these SU(2) symmetries will never be perfectly preserved due to the presence of thermal effects and lattice imperfections. In this case, assuming these unwanted mechanisms are sufficiently small in magnitude compared to the driving strength, then we expect the correlated states to instead form transiently and be observable on some intermediate timescale, prior to the system eventually heating up to a featureless infinite temperature states.

\section{CONCLUSION}

In this paper we have simultaneously diagonalized the dual SU(2) symmetries of the Hubbard model on an arbitrary graph. This diagonalization has allowed us to construct the long-time states of the driven model and classify and predict their properties under various symmetry classes of driving. The preservation of either, or both, of the SU(2) symmetries leads to a significant dynamical reordering of the long-range correlations of the system, resulting in states with off-diagonal long-range correlations in the corresponding symmetry sectors. We have analyzed how these correlations scale with the relevant initial state properties, lattice filling, and the graph size. This analysis led us to identify a mechanism by which a unique condensate in the thermodynamic limit—hosting both spin-exchange and particle-hole order simultaneously-can be formed.

Here, we have focused directly on the case of periodic driving, where a many-body system will generically undergo the desired heating for most nontrivial driving terms and experimental implementations are possible with current technologies. We emphasize, however, that this mechanism of 'heating-induced order' can occur in any quantum system which continuously absorbs energy from an external source while the requisite symmetries are preserved. For example, coupling the system to an energetic Markovian external source which introduces decoherence through local, hermitian jump operators has been shown to cause the desired heating [40,52], and driving in the form of strong kicking or nonmonochromatic pulses $[51,53,54]$ is likely to also provide a route to inducing these correlated states.

Alongside this, our work opens up several further questions. Firstly, we have not quantified the effect of the polygon symmetries of the Hubbard lattice on the long-time states reached under periodic driving. While there is a minimal effect on the geometries we consider, more complicated structures could significantly alter the pairing landscape of the system, revealing exotic states which have preferential directions for the flow of supercurrents within the lattice structure.

Furthermore we emphasize that this intuition of symmetry-constrained relaxation to ordered states is not limited to the single-band Hubbard model. For example, the $n$ species Hubbard model is SU(n) symmetric [55] and driving terms which preserve this symmetry would lead to a unique state which possesses off-diagonal long-range spin-exchange correlations between each distinct pair of fermionic species. Moreover, a number of other systems, such as the SU(n) Heisenberg model [56] or multiband/multiorbital Hubbard models [57,58], possess special unitary/orthogonal symmetries which could be exploited to realize similar exotic correlated states under heating.

\section{ACKNOWLEDGMENTS}

We would like to thank Martin Claassen, Yao Wang, Andrea Cavalleri, Michelle Buzzi, and Daniele Nicoletti for helpful comments. This work has been supported by EPSRC Grant Nos. EP/P009565/1 and EP/K038311/1 and is partially funded by the European Research Council under 
the European Unions Seventh Framework Programme (FP7/2007-2013)/ERC Grant Agreement No. 319286 QMAC. J.T. is also supported by funding from Simon Harrison. M.A.S. acknowledges support by the DFG through the Emmy
Noether programme (SE 2558/2-1) and F.S. acknowledges support from the Cluster of Excellence 'Advanced Imaging of Matter' of the Deutsche Forschungsgemeinschaft (DFG) EXC 2056 - project ID 390715994.
[1] H. Levine, A. Keesling, G. Semeghini, A. Omran, T. T. Wang, S. Ebadi, H. Bernien, M. Greiner, V. Vuletić, H. Pichler, and M. D. Lukin, Parallel Implementation of High-Fidelity Multiqubit Gates with Neutral Atoms, Phys. Rev. Lett. 123, 170503 (2019).

[2] A. Zenesini, H. Lignier, D. Ciampini, O. Morsch, and E. Arimondo, Coherent Control of Dressed Matter Waves, Phys. Rev. Lett. 102, 100403 (2009).

[3] M. Mitrano, A. Cantaluppi, D. Nicoletti, S. Kaiser, A. Perucchi, S. Lupi, P. Di Pietro, D. Pontiroli, M. Riccò, S. Clark, D. Jaksch, and A. Cavalleri, Possible light-induced superconductivity in $\mathrm{K}_{3} \mathrm{C}_{60}$ at high temperature, Nature (London) 530, 461 (2016).

[4] D. Fausti, R. I. Tobey, N. Dean, S. Kaiser, A. Dienst, M. C. Hoffmann, S. Pyon, T. Takayama, H. Takagi, and A. Cavalleri, Light-induced superconductivity in a stripe-ordered cuprate, Science 331, 189 (2011).

[5] W. Hu, S. Kaiser, D. Nicoletti, C. R. Hunt, I. Gierz, M. C. Hoffmann, M. Le Tacon, T. Loew, B. Keimer, and A. Cavalleri, Optically enhanced coherent transport in $\mathrm{YBa}_{2} \mathrm{Cu}_{3} \mathrm{O}_{6.5}$ by ultrafast redistribution of interlayer coupling, Nat. Mater. 13, 705 (2014).

[6] D. Nicoletti, E. Casandruc, Y. Laplace, V. Khanna, C. R. Hunt, S. Kaiser, S. S. Dhesi, G. D. Gu, J. P. Hill, and A. Cavalleri, Optically induced superconductivity in striped $\mathrm{La}_{2-x} \mathrm{Ba}_{x} \mathrm{CuO}_{4}$ by polarization-selective excitation in the near infrared, Phys. Rev. B 90, 100503(R) (2014).

[7] M. Budden, T. Gebert, M. Buzzi, G. Jotzu, E. Wang, T. Matsuyama, G. Meier, Y. Laplace, D. Pontiroli, M. Riccò, F. Schlawin, D. Jaksch, and A. Cavalleri, Evidence for metastable photo-induced superconductivity in $\mathrm{K}_{3} \mathrm{C}_{60}$, arXiv:2002.12835.

[8] K. A. Cremin, J. Zhang, C. C. Homes, G. D. Gu, Z. Sun, M. M. Fogler, A. J. Millis, D. N. Basov, and R. D. Averitt, Photoenhanced metastable c-axis electrodynamics in stripe-ordered cuprate $\mathrm{La}_{1.885} \mathrm{Ba}_{0.115} \mathrm{CuO}_{4}$, Proc. Natl. Acad. Sci. USA 116, 19875 (2019).

[9] A. Cantaluppi, M. Buzzi, G. Jotzu, D. Nicoletti, M. Mitrano, D. Pontiroli, M. Riccò, A. Perucchi, P. Di Pietro, and A. Cavalleri, Pressure tuning of light-induced superconductivity in $\mathrm{K}_{3} \mathrm{C}_{60}$, Nat. Phys. 14, 837 (2018).

[10] D. Nicoletti and A. Cavalleri, Nonlinear light-matter interaction at terahertz frequencies, Adv. Opt. Photonics 8, 401 (2016).

[11] M. Buzzi, D. Nicoletti, M. Fechner, N. Tancogne-Dejean, M. A. Sentef, A. Georges, T. Biesner, E. Uykur, M. Dressel, A. Henderson, T. Siegrist, J. A. Schlueter, K. Miyagawa, K. Kanoda, M.-S. Nam, A. Ardavan, J. Coulthard, J. Tindall, F. Schlawin, D. Jaksch, and A. Cavalleri, Photomolecular High-Temperature Superconductivity, Phys. Rev. X 10, 031028 (2020).

[12] J. R. Coulthard, S. R. Clark, S. Al-Assam, A. Cavalleri, and D. Jaksch, Enhancement of superexchange pairing in the periodically driven Hubbard model, Phys. Rev. B 96, 085104 (2017)
[13] M. Knap, M. Babadi, G. Refael, I. Martin, and E. Demler, Dynamical Cooper pairing in nonequilibrium electron-phonon systems, Phys. Rev. B 94, 214504 (2016).

[14] T. Kaneko, T. Shirakawa, S. Sorella, and S. Yunoki, Photoinduced $\eta$ Pairing in the Hubbard Model, Phys. Rev. Lett. 122, 077002 (2019).

[15] M. A. Sentef, A. Tokuno, A. Georges, and C. Kollath, Theory of Laser-Controlled Competing Superconducting and Charge Orders, Phys. Rev. Lett. 118, 087002 (2017).

[16] D. M. Kennes, E. Y. Wilner, D. R. Reichman, and A. J. Millis, Transient superconductivity from electronic squeezing of optically pumped phonons, Nat. Phys. 13, 479 (2017).

[17] M. Babadi, M. Knap, I. Martin, G. Refael, and E. Demler, Theory of parametrically amplified electron-phonon superconductivity, Phys. Rev. B 96, 014512 (2017).

[18] T. Kaneko, S. Yunoki, and A. J. Millis, Charge stiffness and long-range correlation in the optically induced $\eta$-pairing state of the one-dimensional Hubbard model, Phys. Rev. Research 2, 032027(R) (2020).

[19] A. Sheikhan and C. Kollath, Dynamically enhanced unconventional superconducting correlations in a Hubbard ladder, Phys. Rev. B 102, 035163 (2020).

[20] Y. Wang, C.-C. Chen, B. Moritz, and T. P. Devereaux, Light-Enhanced Spin Fluctuations and $d$-Wave Superconductivity at a Phase Boundary, Phys. Rev. Lett. 120, 246402 (2018).

[21] D. M. Kennes, M. Claassen, M. A. Sentef, and C. Karrasch, Light-induced $d$-wave superconductivity through Floquetengineered Fermi surfaces in cuprates, Phys. Rev. B 100, 075115 (2019).

[22] Z. M. Raines, V. Stanev, and V. M. Galitski, Enhancement of superconductivity via periodic modulation in a three-dimensional model of cuprates, Phys. Rev. B 91, 184506 (2015).

[23] Y. Murakami, N. Tsuji, M. Eckstein, and P. Werner, Nonequilibrium steady states and transient dynamics of conventional superconductors under phonon driving, Phys. Rev. B 96, 045125 (2017).

[24] M. A. Sentef, Light-enhanced electron-phonon coupling from nonlinear electron-phonon coupling, Phys. Rev. B 95, 205111 (2017).

[25] M. W. Cook and S. R. Clark, Controllable finite-momenta dynamical quasicondensation in the periodically driven onedimensional Fermi-Hubbard model, Phys. Rev. A 101, 033604 (2020).

[26] C. Rylands, E. B. Rozenbaum, V. Galitski, and R. Konik, ManyBody Dynamical Localization in a Kicked Lieb-Liniger Gas, Phys. Rev. Lett. 124, 155302 (2020).

[27] H. Gao, J. R. Coulthard, D. Jaksch, and J. Mur-Petit, Anomalous Spin-Charge Separation in a Driven Hubbard System, Phys. Rev. Lett. 125, 195301 (2020).

[28] R. Versteeg, A. Chiocchetta, F. Sekiguchi, A. Aldea, A. Sahasrabudhe, K. Budzinauskas, Z. Wang, V. Tsurkan, A. Loidl, 
D. Khomskii, S. Diehl, and P. Loosdrecht, Nonequilibrium quasistationary spin disordered state in the Kitaev-Heisenberg magnet $\alpha-\mathrm{RuCl}_{3}$, arXiv:2005.14189.

[29] L. D'Alessio and M. Rigol, Long-Time Behavior of Isolated Periodically Driven Interacting Lattice Systems, Phys. Rev. X 4, 041048 (2014).

[30] A. Lazarides, A. Das, and R. Moessner, Equilibrium states of generic quantum systems subject to periodic driving, Phys. Rev. E 90, 012110 (2014).

[31] M. Bukov, S. Gopalakrishnan, M. Knap, and E. Demler, Prethermal Floquet Steady States and Instabilities in the Periodically Driven, Weakly Interacting Bose-Hubbard Model, Phys. Rev. Lett. 115, 205301 (2015).

[32] E. Canovi, M. Kollar, and M. Eckstein, Stroboscopic prethermalization in weakly interacting periodically driven systems, Phys. Rev. E 93, 012130 (2016).

[33] D. A. Abanin, W. De Roeck, and F. Huveneers, Exponentially Slow Heating in Periodically Driven Many-Body Systems, Phys. Rev. Lett. 115, 256803 (2015).

[34] F. Peronaci, M. Schiró, and O. Parcollet, Resonant Thermalization of Periodically Driven Strongly Correlated Electrons, Phys. Rev. Lett. 120, 197601 (2018).

[35] M. Reitter, J. Näger, K. Wintersperger, C. Sträter, I. Bloch, A. Eckardt, and U. Schneider, Interaction Dependent Heating and Atom Loss in a Periodically Driven Optical Lattice, Phys. Rev. Lett. 119, 200402 (2017).

[36] S. A. Weidinger and M. Knap, Floquet prethermalization and regimes of heating in a periodically driven, interacting quantum system, Sci. Rep. 7, 45382 (2017).

[37] K. Singh, C. J. Fujiwara, Z. A. Geiger, E. Q. Simmons, M. Lipatov, A. Cao, P. Dotti, S. V. Rajagopal, R. Senaratne, T. Shimasaki, M. Heyl, A. Eckardt, and D. M. Weld, Quantifying and Controlling Prethermal Nonergodicity in Interacting Floquet Matter, Phys. Rev. X 9, 041021 (2019).

[38] S. Mathey and S. Diehl, Dynamic renormalization group theory for open Floquet systems, Phys. Rev. B 102, 134307 (2020).

[39] B. Buca, J. Tindall, and D. Jaksch, Non-stationary coherent quantum many-body dynamics through dissipation, Nat. Commun. 10, 1730 (2019).

[40] J. Tindall, B. Buča, J. R. Coulthard, and D. Jaksch, HeatingInduced Long-Range $\eta$ Pairing in the Hubbard Model, Phys. Rev. Lett. 123, 030603 (2019).

[41] F. H. L. Essler, H. Frahm, F. Ghmann, A. Klmper, and V. E. Korepin, The One-Dimensional Hubbard Model (Cambridge University Press, Cambridge, United Kingdom, 2005).

[42] P. Woit, Quantum Theory, Groups and Representations: An Introduction (Springer International Publishing, New York, USA, 2017).

[43] A. Barut and R. Rkaczka, Theory of Group Representations and Applications (Polish Scientific Publishers, Warsaw, Poland, 1980).
[44] D. F. Bailey, Counting arrangements of 1's and -1's, Mathematics Magazine 69, 128 (1996).

[45] C. N. Yang, Concept of off-diagonal long-range order and the quantum phases of liquid He and of superconductors, Rev. Mod. Phys. 34, 694 (1962).

[46] H. T. Nieh, G. Su, and B.-H. Zhao, Off-diagonal long-range order: Meissner effect and flux quantization, Phys. Rev. B 51, 3760 (1995).

[47] R. A. Hart, P. M. Duarte, T.-L. Yang, X. Liu, T. Paiva, E. Khatami, R. T. Scalettar, N. Trivedi, D. A. Huse, and R. G. Hulet, Observation of antiferromagnetic correlations in the Hubbard model with ultracold atoms, Nature (London) 519, 211 (2015).

[48] L. Tarruell and L. Sanchez-Palencia, Quantum simulation of the Hubbard model with ultracold fermions in optical lattices, C. R. Phys. 19, 365 (2018).

[49] M. Köhl, H. Moritz, T. Stöferle, K. Günter, and T. Esslinger, Fermionic Atoms in a Three Dimensional Optical Lattice: Observing Fermi Surfaces, Dynamics, and Interactions, Phys. Rev. Lett. 94, 080403 (2005).

[50] M. Messer, K. Sandholzer, F. Görg, J. Minguzzi, R. Desbuquois, and T. Esslinger, Floquet Dynamics in Driven Fermi-Hubbard Systems, Phys. Rev. Lett. 121, 233603 (2018).

[51] J. Tindall, F. Schlawin, M. Buzzi, D. Nicoletti, J. R. Coulthard, H. Gao, A. Cavalleri, M. A. Sentef, and D. Jaksch, Dynamical Order and Superconductivity in a Frustrated Many-Body System, Phys. Rev. Lett. 125, 137001 (2020).

[52] J. Tindall, C. S. Muñoz, B. Buča, and D. Jaksch, Quantum synchronisation enabled by dynamical symmetries and dissipation, New J. Phys. 22, 013026 (2020).

[53] Y. Wang, M. Claassen, B. Moritz, and T. P. Devereaux, Producing coherent excitations in pumped Mott antiferromagnetic insulators, Phys. Rev. B 96, 235142 (2017).

[54] Y. Wang, T. P. Devereaux, and C.-C. Chen, Theory of timeresolved Raman scattering in correlated systems: Ultrafast engineering of spin dynamics and detection of thermalization, Phys. Rev. B 98, 245106 (2018).

[55] W. Nie, D. Zhang, and W. Zhang, Ferromagnetic ground state of the su(3) Hubbard model on the Lieb lattice, Phys. Rev. A 96, 053616 (2017).

[56] K. S. D. Beach, F. Alet, M. Mambrini, and S. Capponi, $\mathrm{SU}(n)$ Heisenberg model on the square lattice: A continuous$n$ quantum Monte Carlo study, Phys. Rev. B 80, 184401 (2009).

[57] Z.-J. Ying, Y.-Q. Li, and S.-J. Gu, Symmetries in the Hubbard model with n-fold orbital degeneracy, J. Math. Phys. 42, 4865 (2001).

[58] Y. Wang, J. P. Dehollain, F. Liu, U. Mukhopadhyay, M. S. Rudner, L. M. K. Vandersypen, and E. Demler, Ab initio exact diagonalization simulation of the Nagaoka transition in quantum dots, Phys. Rev. B 100, 155133 (2019). 